Classification

Physics Abstracts

$74.50-74.60 \mathrm{~J}$

\title{
Critical transport currents of superconducting microarrays
}

\author{
H. J. Fink (*) and A. López \\ Centro Atómico Bariloche, Comisión Nacional de Energía Atómica, 8400 Bariloche, Argentina
}

(Reçu le 19 avril 1985, révisé le 12 août, accepté le 29 août 1985)

\begin{abstract}
Résumé. - Nous avons déterminé les densités critiques de courant supraconducteur à partir des équations de Ginzburg-Landau non linéaires pour des courants de transport injectés dans un réseau carré, en fonction de la période du réseau. A la limite où celle-ci devient petite devant la longueur de cohérence supraconductrice, on a trouvé aussi des valeurs maximales du courant pour les réseaux rectangulaires et hexagonaux. Pour ces derniers les intensités de courant critique sont anisotropes avec symétrie d'ordre 3 dans le plan du réseau.
\end{abstract}

\begin{abstract}
From the nonlinear Ginzburg-Landau equations exact maximum supercurrent densities of injected transport currents were calculated for a square microarray as a function of lattice parameter. In the limit that the lattice parameter becomes small in comparison to the temperature dependent coherence length, values of the maximum current were also obtained for honeycomb and rectangular arrays. The honeycomb array has anisotropic critical current densities with 3-fold symmetry in the plane of the array.
\end{abstract}

Theoretical predictions concerning the properties of superconducting micronetworks [1-3] have recently spurred a number of experimental studies $[4,5]$. Second order phase transitions in the presence of a magnetic field, described by the theories of Alexander, de Gennes and others, should ideally be investigated experimentally via magnetic susceptibility measurements. In practice, however, several resistance studies of the second order phase boundary of micronetworks have been made which involve current transitions. This applies also to experimental studies on arrays with Josephson junctions [6].

To bring the theory into closer relation with experiment it is important to understand current [7] induced transitions correctly, and for this the nonlinear Ginzburg-Landau equations must be used. Now, already from the linearized theory we know that an applied external magnetic field will generate inhomogeneous [8] current distributions, which at the moment are intractable in the context of the full GL formulation.

For this reason we have limited ourselves here to the study of a few chosen arrays in zero external field. Their importance lies in the fact that for these systems we can make reasonable intuitive assumptions concerning the flow of an externally injected current through them.

$\left(^{*}\right)$ Permanent address : Department of Electrical and Computer Engineering, University of California, Davis, CA 95616, U.S.A. 
We consider first an infinite square lattice of spacing $L$ (Fig. 1a). We assume that the injected current flows only along the horizontal $(x)$ branches in the absence of an applied magnetic field.

Below the superconducting transition temperature, the presence of a current along $x$ will depress the order parameter near the nodes on the vertical $(y)$ branches. If end effects are neglected, symmetry considerations allow us to reduce this problem to that of an " elementary cell " such as $A_{1} A_{2} B_{1} B_{2}$ in figure 1a. At points $A_{1}$ and $A_{2}$, midway between two nodes on the current carrying branches, the order parameter is a minimum, while it is a maximum at $B_{1}$ and $B_{2}$ (midpoints of the $y$ branches with zero current). If the thickness of the wires is smaller than the temperature dependent coherence length $\xi(t)$, we may use the following one-dimensional GL equation for the order parameter

$$
\frac{\mathrm{d}^{2} f}{\mathrm{~d} x^{2}}+\left[1-f^{2}-\frac{I^{2}}{f^{4}}\right] f=0
$$

Here $f=\left[\psi / \psi_{\infty}\right]$ is the modulus of the normalized GL order parameter and $\psi_{\infty}$ its value at zero field and zero current. The normalized current density is $I=(4 \pi / c)\left(2 \pi \xi \lambda^{2} / \phi_{0}\right) J$, where $J$ is the current density in cgs Gaussian units, $\phi_{0}$ is the fluxoid quantum and $\lambda$ is the magnetic field penetration depth. The curvilinear coordinate $x$ is normalized by $\xi(t)$. For a long wire without side branches it follows from equation (1) that the critical current $I_{\mathrm{c}}$ is $I_{\mathrm{c}}(\infty)=2 / \sqrt{27}$.

Equation (1) has a first integral

$$
2 f^{2}\left(\frac{\mathrm{d} f}{\mathrm{~d} x}\right)^{2}=\left(f_{0}^{2}-f^{2}\right)\left[f^{2}\left(2-f_{0}^{2}-f^{2}\right)-\frac{2 I^{2}}{f_{0}^{2}}\right]
$$

where $f_{0}$ is the value of $f$ at an extremal point $x_{0}$ at which $\mathrm{d} f / \mathrm{d} x=0$. Defining $t^{2}(x)=f^{2}(x)-f_{0}^{2}$

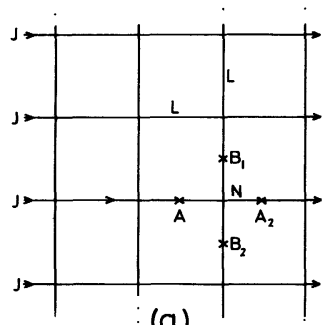

(a)
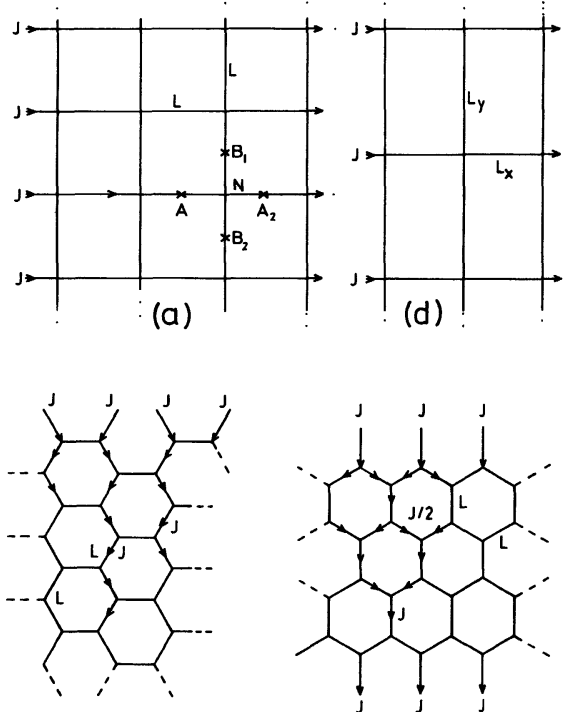

(b)

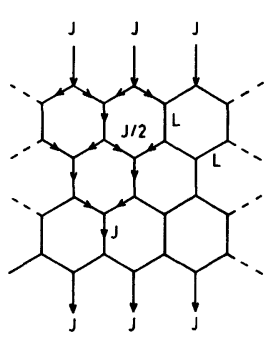

(c)

Fig. 1. - Shown are various arrays with injected transport currents $J$ with no applied magnetic field : (a) square, (b) and (c) honeycomb and (d) rectangular array. The building block $A_{1} B_{1} A_{2} B_{2}$ around the node $\mathbf{N}$ is shown for the square array. 
equation (2) becomes

where

$$
2\left(\frac{\mathrm{d} t}{\mathrm{~d} x}\right)^{2}=\left(t^{2}-R_{1}\right)\left(t^{2}-R_{2}\right)
$$

$$
R_{1,2}=\left(1-\frac{3 f_{0}^{2}}{2}\right) \pm\left[\left(1-\frac{f_{0}^{2}}{2}\right)^{2}-\frac{2 I^{2}}{f_{0}^{2}}\right]^{1 / 2}
$$

The solution of equation (3) is a Jacobian elliptic function which depends on $R_{1}$ and $R_{2}$.

At each node complex current conservation applies giving the well known relation [3]

$$
\sum_{n}\left(i \hbar \frac{\partial \psi_{n}}{\partial x}+\left(\frac{2 e}{c}\right) A \psi_{n}\right)=0
$$

where the sum is over all branches joining at a given node. If we write $\psi=f \mathrm{e}^{i \phi}$, the real and imaginary parts of (5) are

$$
\begin{gathered}
\sum_{n}\left(\frac{\mathrm{d} \phi}{\mathrm{d} x}-\frac{2 \pi A}{\phi_{0}}\right)=\sum_{n} q_{n}=0 \\
\sum_{n} \frac{\mathrm{d} f}{\mathrm{~d} x}=0 .
\end{gathered}
$$

Here $q_{n}$ is the superfluid velocity, $A$ the vector potential and the derivatives are taken radially outward from the node.

In an initial state of zero current, the order parameter $f$ is unity uniformly throughout the lattice. As the applied current grows, the order parameter will start to decrease, becoming depressed at a faster rate in the current carrying branches.

Using the known Jacobian elliptic function solutions of equation (3), a numerical search was made for values of the order parameter which would satisfy simultaneously the above conditions (6a) and (6b) and the continuity of $f_{\mathrm{N}}$ on both vertical and horizontal branches at the nodes.

We show in figure 2 the results for the reduced cell $A_{1} A_{2} B_{1} B_{2} N$ of figure 1a, giving the

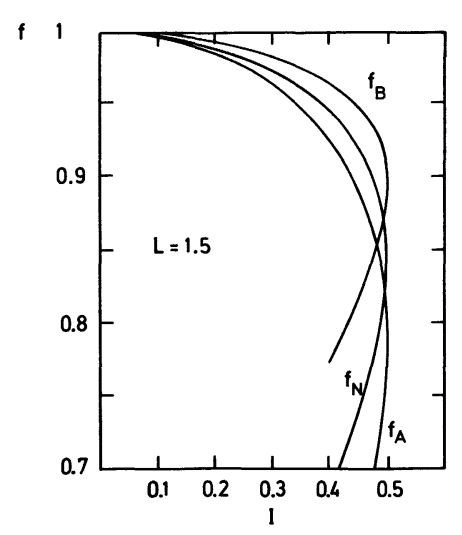

Fig. 2. - The values of the order parameter at positions $\mathrm{A}_{1}$ and $\mathrm{A}_{2}, f_{\mathrm{A}}$, at $\mathrm{N}, f_{\mathrm{N}}$, and at $\mathrm{B}_{1}$ and $\mathrm{B}_{2}, f_{\mathrm{B}}$, as indicated in figure 1a, are shown as a function of the normalized current density for a square array of lattice parameter $L=1.5 \xi(t)$. 
dependence of the order parameter on the current density at $f_{\mathrm{A}}, f_{\mathrm{B}}$ and $f_{\mathrm{N}}$ (maximum, minimum and node) for a nodal spacing $L=1.5 \xi$. It is seen that the order parameter decreases with increasing current until a critical current is reached, at which point a transition to (presumably) the normal state takes place. The physically meaningful region is given by $\mathrm{d} f / \mathrm{d} j \leqslant 0$. All three curves reach the maximum at the same value of the critical current density, which is thus uniquely defined. Similar curves were obtained for different nodal spacings.

Figure 3 shows the dependence of the maximum (critical) current density on the normalized lattice parameter $L / \xi(t)$. We have plotted $I_{\mathrm{c}} v s$. $(L / \xi(t))^{2}$ which is proportional to $\left(1-T / T_{\mathrm{c}}\right)$ close to the transition temperature $T_{\mathrm{c}}$.

For $L$ values much larger than $\xi, I_{\mathrm{c}}$ tends to the limiting value $2 / \sqrt{27}$ of the bare wire, while for small values of $L$ it approaches $2 \sqrt{2 / 27}$. The latter result [9] can be obtained by a series expansion of equation (3) of the form $t(x)=a_{1} x+a_{3} x^{3}$.

The limiting values for the honeycomb lattice can be obtained in a similar fashion. In this case, due to the threefold symmetry, the critical current depends on orientation varying between a minimum and a maximum value (case 1 and case 2 in figures $1 \mathrm{~b}$ and $1 \mathrm{c}$ ).

The limiting values for $L / \xi \rightarrow 0$ are $I_{\mathrm{c}}(1)=\sqrt{3 / 2} I_{\mathrm{c}}(\infty)$ and $I_{\mathrm{c}}(2)=\sqrt{2} I_{\mathrm{c}}(\infty)$ which is the same as for the square lattice.

For a rectangular lattice of spacings $L_{x}$ and $L_{y}$, with $L_{x} / L_{y}$ fixed and current directed along $x$ (Fig. 1d), the critical current for small lattice spacings is $I_{c}=\sqrt{1+L_{y} / L_{x}} I_{c}(\infty)$. When the lattice spacing of any two dimensional array becomes very large, it can easily be seen that the critical current is the same, for all cases, namely that of the infinite wire $I_{c}(\infty)=2 / \sqrt{27}$.

In conclusion, we have obtained from the nonlinear GL equations exact results for the maximum supercurrent density as a function of normalized lattice spacing (or temperature) in zero applied magnetic field. In the limit that the lattice spacing becomes small compared to $\xi(t)$, values of critical currents for other geometric arrays were obtained. In particular, it was found that the honeycomb lattice has anisotropic critical current densities with 3-fold symmetry in the plane of the array.

\section{Acknowledgments.}

We thank V. Grünfeld for her constructive help with the manuscript and Stephen Roberts and Joey Loo for aiding with the numerical work.

One of the authors (H. J. F.) was supported in part by NSF Grant INT 8213132 while on leave from the University of California.

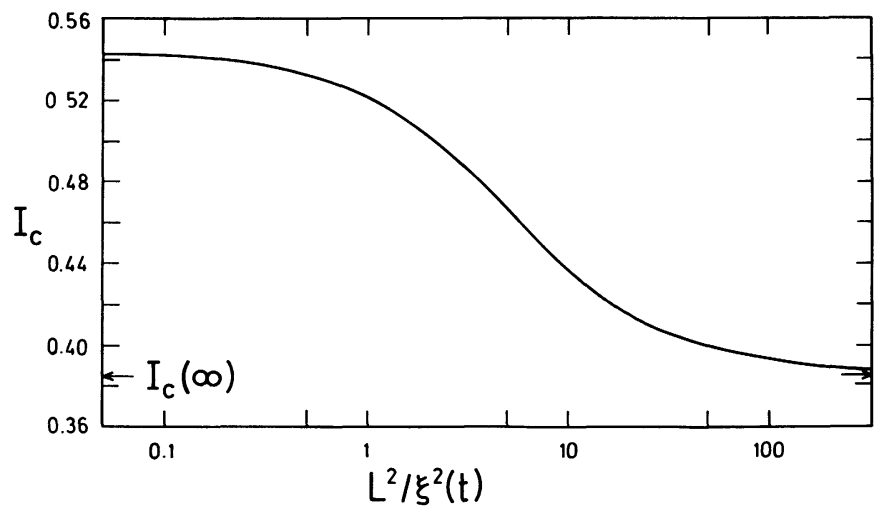

Fig. 3. - The maximum (critical) value of the current density, as shown in figure 2, is plotted as a function of the square of the normalized lattice parameter for a square lattice. The abcissa is proportional to $1-T / T_{\mathrm{c}}$. 


\section{References}

[1] De Gennes, P. G., C. R. Acad. Sci. Paris, Ser. II 292 (1981) 279.

[2] AleXander, S., Phys. Rev. B 27 (1983) 1541.

[3] Fink, H. J., López, A. and Maynard, R., Phys. Rev. B 26 (1982) 5237.

[4] Pannetier, B., Chaussy, J. and Rammal, R., J. Physique Lett. 44 (1983) L-853.

[5] Pannetier, B., Wang, Y. Y. and Chaussy, J., Proc. 17-th Int. Conf. Low Temp. Phys., Eds. : U. Eckern, A. Schmid, W. Weber and H. Wühl (North-Holland) 1984, p. 735.

[6] LoBb, C. J., Physica B +C 126 (1984) 319 and references therein.

[7] Fink, H. J. and Grünfeld, V., Proc. 17-th Int. Conf. Low Temp. Phys., Eds. : U. Eckern, A. Schmid, W. Weber and H. Wühl (North-Holland) 1984, p. 721.

[8] Simonin, J., Rodrigues, D. and López, A., Phys. Rev. Lett. 49 (1982) 944.

[9] Also obtained by PANNETIER, B., private communication to V. Grünfeld. 